\title{
Effects of salts on pre-crystalline lysozyme aggregation characterized by forward static light scattering
}

\author{
Takashi Wakamatsu* \\ National Institute of Technology, Ibaraki College, Hitachinaka, Ibaraki, 312-8508, Japan \\ * Corresponding author: Fax: 81-29-271-2930, and/or e-mail: wakamatu@ee.ibaraki-ct.ac.jp
}

\begin{abstract}
We present the aggregation effects of the precipitant chloride salts with different cations, namely $\mathrm{KCl}, \mathrm{MgCl}_{2}$ and $\mathrm{CaCl}_{2}$, on hen egg-white lysozyme (HEWL) crystallization, characterized by forward static light scattering at small angles. The pre-crystalline HEWL solutions with the chloride salts exhibit non-integer power-law profiles of forward light scatterings, indicating formation of fractal aggregates. The fractal aggregates with a closely packed structure were formed in the solutions with the chloride salts under crystallization conditions. The crystallization ability ranking of the cations almost agrees with the Hofmeister series. $\mathrm{CaCl}_{2}$ presented the slowest crystal growth of HEWL, owing to the weakest effect of aggregation on HEWL among the chloride salts studied.
\end{abstract}

Key words: protein crystallization, lysozyme, chloride salt, light scattering, protein aggregation

\section{INTRODUCTION}

Fabrication of protein crystals with good quality based on $\mathrm{x}$-ray or neutron diffraction analysis is a key challenge in protein crystallography. Protein crystallizations remain reliant on trial and error procedures $[1,2]$. In crystallizing solutions of the typical protein lysozyme, the addition of electrolytic salts induces a liquid-liquid phase separation in a saturated area of the phase diagram and the lysozyme crystals grow in lysozyme-rich droplets [2, 3]. The mechanism by which electrolytic ions solvate lysozymes in solutions, however, remains unclear. In protein crystallization, characterization of protein aggregates of submicron size in the pre-crystalline stage provides essential information for understanding the protein crystallization process $[4,5]$. Whereas effects of electrolytic salts on the solubility of aqua proteins are known as the Hofmeister series [6, 7], which are strongly associated with protein aggregation, the series is empirical and the origin has remained unclear since the 19th century.

Forward light scattering (FLS) at small angles is an optical response of material aggregates formed from a dissolving solution, such as proteins strongly associated with surrounding solvent molecules [8,9]. Several studies have revealed via dynamic light scattering (DLS) $[10,11]$ and static light scattering (SLS) $[12,13]$ at low angles of around $20-30^{\circ}$ that fractal aggregates are formed in precrystalline lysozyme solutions. Previous work [14, 15] based on forward static-light-scattering (F-SLS) measurements presents that fractal aggregates of hen eggwhite lysozymes (HEWL) derived by precipitant $\mathrm{NaCl}$ have a dense structure (the fractal dimension $D>1.5$ ) in solutions progressing towards crystallization. However, relationships between aggregation and crystallization of HEWL induced by salts other than $\mathrm{NaCl}$ have not been established. Here we evaluated aggregation effects induced by other chloride salts, namely $\mathrm{KCl}, \mathrm{MgCl}_{2}$ and $\mathrm{CaCl}_{2}$ and compared them with those of $\mathrm{NaCl}$ addition, by using the F-SLS technique. We discussed the relationship between the fractal aggregation and the crystallization conditions for the different cations of the chloride salts.

\section{MATERIALS AND METHOD \\ 2.1 Sample materials}

HEWL (Seikagaku Co., six times recrystallized) were used for aggregation and crystallization studies without further purification. HEWL is an aqueous protein with a molecular weight of about $14 \mathrm{kDa}$ and its monomer has a nearly ellipsoidal shape. The solutions required for this study were prepared separately at $20 \pm 0.5^{\circ} \mathrm{C}$ : a lysozyme solution of $90 \mathrm{mg} / \mathrm{ml}(6.3 \mathrm{mM})$ in a $50 \mathrm{mM}$ sodium acetate buffer $(p \mathrm{H}=4.6)$, and salt solutions of $15 \%(\mathrm{w} / \mathrm{v})$ for $\mathrm{KCl}, \mathrm{MgCl}_{2}$ and $\mathrm{CaCl}_{2}$ in the same sodium acetate buffer, as stock solutions. The salt concentrations of $15 \%$ (w/v) for $\mathrm{KCl}, \mathrm{MgCl}_{2}$ and $\mathrm{CaCl}_{2}$ correspond to $2.01 \mathrm{M}$, $1.59 \mathrm{M}$ and $1.35 \mathrm{M}$ respectively. These solutions were passed through a filter with a pore size of $0.2 \mu \mathrm{m}$ to remove any dust from the solutions. The lysozyme crystallization experiments were performed using a batch method. Immediately before the crystallization or the FSLS measurements, the HEWL, the salt solutions and the buffer were combined at an appropriate rate in a micro test tube $(1.5 \mathrm{ml})$ at $20 \pm 0.5^{\circ} \mathrm{C}$. The mixture sample ( $\sim 22$ $\mu \mathrm{l})$ for the F-SLS measurements were taken immediately from the test tube and injected into a sample cell.

2.2 Fractal aggregates and forward static light scattering When a light beam is irradiated on a target, an inhomogeneous distribution of local dielectric function $\varepsilon(\boldsymbol{r})$ induces SLS that has a characteristic scatteringangle-dependent pattern. The spatial profiles of SLS, $I_{\mathrm{s}}(q)$, expressed as a function of the scattering vector $q$, provide information of the internal structure of scatters such as aggregates. The $q$ is given by $4 \pi n / \lambda \sin \left(\theta_{\mathrm{s}} / 2\right)$, where $n$ is the refractive index of the surrounding medium and $\theta_{\mathrm{s}}$ is 
the scattering angle. $I_{\mathrm{s}}(q)$ linearly involves a structure factor $S(q)$ that is given by the Fourier transform of the average local density $\left\langle\rho(r)>_{0}\right.$ directly related to a density correlation function in the scatters [16]. The local density $\rho(r)$ is defined as the number (mass) density of a minimum unit like a monomer at the position $r$ in the scatters, and is directly correlated to $\varepsilon(\boldsymbol{r})$. Fractal aggregates are typically aggregates in the pre-crystalline stage, and are characterized by a power-law dependence of the averaged local density [16]:

$$
<\rho(r)>_{0}=c r^{D-d} \quad(D<d),
$$

where $c$ is a constant independent of $r$ and $d$ is the spatial dimension (e.g., $d=3$ for the aggregates in the solutions). As a result, $I_{\mathrm{s}}(q)$ patterns attributed to the fractal aggregates also exhibit a power-law relationship with $q$ :

$$
I_{\mathrm{s}}(q)=C q^{-D}
$$

where $C$ is a factor independent of the internal structure of aggregates. Since the measured $I_{\mathrm{s}}(q)$ shows power-law characteristics, expressed by $I_{\mathrm{s}}(q)=I(0) q^{-\alpha}, \alpha>0$, we can obtain the power value $\alpha$ from linear fittings of $\log I_{\mathrm{s}} \mathrm{vs}$. $\log q$, and can estimate the values of the fractal dimension $D$ for fractal aggregates, where $D$ is considered to be equal to $\alpha$.

\subsection{Experimental setup}

The setup for F-SLS measurements has been described in detail elsewhere [15]. The developed apparatus adopted a conventional SLS-measurement technique of photodetector scanning using a gonio-stage to precisely measure the F-SLS profiles. The light used for the measurements was a narrow laser beam from a diodepumped solid laser $(\lambda=473 \mathrm{~nm}, 25 \mathrm{~mW}$, spread angle $<$ $1.2 \mathrm{mrad})$. This apparatus allows precise measurements of F-SLS at small angles ranging from $30^{\circ}$ to $0.5^{\circ}$ with a resolution angle of $0.05^{\circ}$. For example, the scattering angle range of $1.5^{\circ}<\theta_{\mathrm{s}}<15^{\circ}$ corresponds to a scattering wave of $0.348 \mu \mathrm{m}^{-1}<q<3.467 \mu \mathrm{m}^{-1}$ for $\lambda=473 \mathrm{~nm}$ $[14,15]$. A sample cell was composed of two microscope cover glass plates $(B K-7, t=0.15 \mathrm{~mm}, 18 \mathrm{~mm} \times 32 \mathrm{~mm}$ ) and a silicon rubber sheet ( $\sim 1 \mathrm{~mm}$ thick). The rubber sheet had a circular hole with a diameter of about $7 \mathrm{~mm}$, and was sandwiched between the two cover glass plates. The mixed lysozyme solution was accommodated in the hole between the glass plates. The thin layer of cells support precise measurements of F-SLS at small angles from the sample solutions. The F-SLS was measured for the lysozyme solutions when a relatively stable state was reached, about $25 \mathrm{~min}$ from the addition of salts. This was because initial scattering responses of lysozyme solutions were drastic just after adding the precipitant salt solution to the lysozyme solutions. All F-SLS measurements were performed with the setup in an air-conditioned room at $20{ }^{\circ} \mathrm{C}$.

\section{RESULTS AND DISCUSSION}

\subsection{Crystallization conditions of lysozymes}

The crystallization experiments were performed with changing the concentrations of the additive salts from 0.5 to $7.0 \%(\mathrm{w} / \mathrm{v})$ every $0.5 \%(\mathrm{w} / \mathrm{v})$ for the HEWL solutions with the fixed concentrations at $20^{\circ} \mathrm{C}$ by a batch method.
We evaluated whether the lysozyme crystal grew or did not within about two weeks after the salt additions and decided the boundary conditions of the salt concentration for the crystallization. Judgment of lysozyme crystallization initiated by the added salts was performed by observing crystal growth in the solutions in microtubes, using a loupe $(\times 10)$ or a microscope $(\times 20-\times 80)$. Crystal growth was observed after 1-4 days from the additions of $\mathrm{KCl}$ or $\mathrm{MgCl}_{2}$. In the case of $\mathrm{CaCl}_{2}$, crystal growth needed 6-10 days, where crystallization of lysozymes was initiated the latest among the three salts. The boundary concentrations of the added chloride salts for crystallization of the $30 \mathrm{mg} / \mathrm{ml}(2.1 \mathrm{mM}) \mathrm{HEWL}$ solution at $20^{\circ} \mathrm{C}$ are summarized in Table I. Here the total ionic strength $I$ at the salt concentrations is also presented, which is frequently used as an index to show the strength relationship between ions in electrolytes: $I=\frac{1}{2} \sum_{i} c_{i} z_{i}^{2}$, where $c_{i}$ is the concentration of ions with the charge valence $z_{i}$. The result for $\mathrm{NaCl}$ in Table I is extracted from Ref. [14] for comparison. Table I shows that under the conditions an ability ranking of the chloride salts for lysozyme crystallization is as follows:

\section{(High) $\quad \mathrm{MgCl}_{2}>\mathrm{CaCl}_{2}>\mathrm{KCl} \sim \mathrm{NaCl}$ (Low ability)}

Considering the valence of the cations however, the crystallization ability ranking of ions is derived from the comparison of ionic strengths of the different cations because the salts have the chloride ion in common. Thus, the ranking is as follows:

(High)

$$
\mathrm{K}^{+} \sim \mathrm{Na}^{+}>\mathrm{Mg}^{2+}>\mathrm{Ca}^{2+}
$$

(Low ability)

Apart from similar ranking between $\mathrm{K}^{+}$and $\mathrm{Na}^{+}$, the crystallization ability ranking aligns with the Hofmeister series [6, 7]: $\mathrm{K}^{+}>\mathrm{Na}^{+}>\mathrm{Mg}^{2+}>\mathrm{Ca}^{2+}$. Here it is worth noting that the Hofmeister series shows an experimentally ranking on precipitation effects of electrolyte ions for aqua proteins, not on protein crystallization effects. The crystallization conditions here indicate that the lysozyme crystallization initiated by the chloride salts is strongly correlated with the precipitation effect.

Table I Comparison of the crystallization boundary for $2.1 \mathrm{mM}$ HEWL with chloride salts at $20^{\circ} \mathrm{C}$ and the ion strengths, involving the result for $\mathrm{NaCl}$.

\begin{tabular}{cccccc}
\hline \multicolumn{2}{c}{ Precipitant salt } & $\mathrm{NaCl}^{\mathrm{a}}$ & $\mathrm{KCl}$ & $\mathrm{MgCl}_{2}$ & $\mathrm{CaCl}_{2}$ \\
\hline \multirow{2}{*}{$c_{i}$} & $\%(\mathrm{w} / \mathrm{v})$ & 3.5 & 4.5 & 3.5 & 5.0 \\
\cline { 2 - 6 } & $\mathrm{M}$ & 0.60 & 0.60 & 0.37 & 0.45 \\
\hline \multirow{2}{*}{$I$} & $\mathrm{M}$ & 0.60 & 0.60 & 1.11 & 1.35 \\
\hline
\end{tabular}

a) Ref. [14]

3.2 Evaluation of the pre-crystalline lysozyme solutions with chloride salts by forward light scattering

Figure 1 shows the measured F-SLS patterns, displayed by $\log I_{\mathrm{s}}-\log q$, for the $30 \mathrm{mg} / \mathrm{ml} \mathrm{HEWL}$ solutions with different chloride salts and without salt for comparison. The F-SLS pattern at low $\mathrm{KCl}$ concentration of $0.27 \mathrm{M}[2.0 \%(\mathrm{w} / \mathrm{v})]$ showed hardly any change compared with that without $\mathrm{KCl}$ in Fig. 1 (a). The F-SLS patterns at 0.54 and $0.81 \mathrm{M} \mathrm{KCl}$ near the crystallization boundary concentration of $0.60 \mathrm{M}[4.5 \%(\mathrm{w} / \mathrm{v})]$ (Table I) showed a power-law type of function. This indicates formation of lysozyme fractal aggregates in the solutions. 
(a)

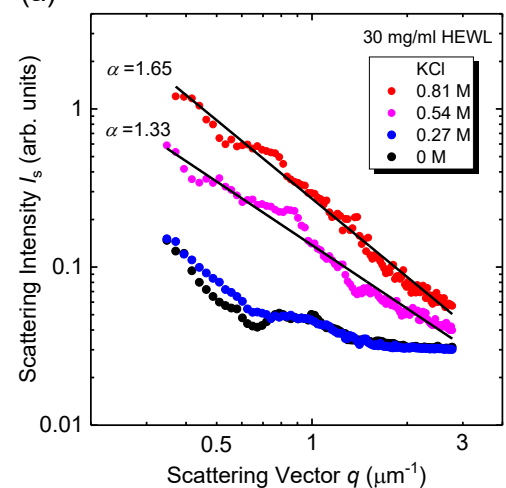

(b)

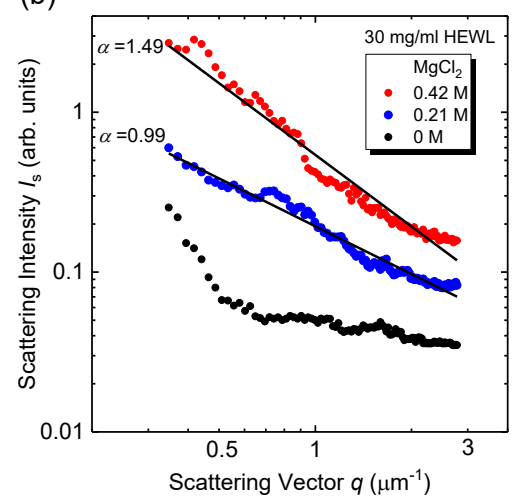

(c)

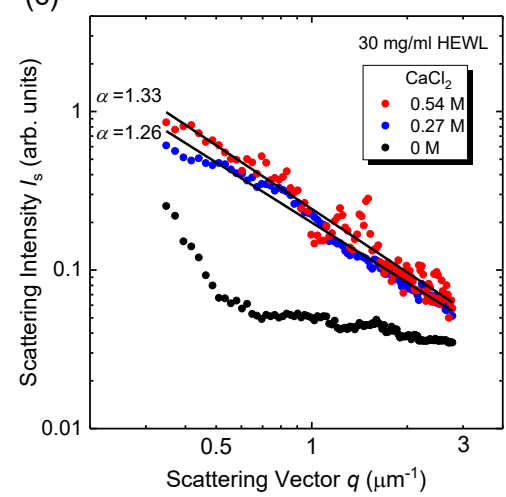

Fig.1 F-SLS profiles (log-log plots) for lysozyme aggregates induced with added chloride salts: (a) $\mathrm{KCl}$, (b) $\mathrm{MgCl}_{2}$ and (c) $\mathrm{CaCl}_{2}$.

The least-square linear fittings for both logarithmic plots of the F-SLS patterns provide the power values $\alpha$ of 1.33 for $0.54 \mathrm{M}$ and 1.65 for $0.81 \mathrm{M} \mathrm{KCl}$. Since a higher fractal dimension value $(D=\alpha)$ is associated with aggregates of a more closely packed structure, the results indicate that the structure of lysozyme aggregates at $0.81 \mathrm{M} \mathrm{KCl}$ is denser than that at $0.54 \mathrm{M} \mathrm{KCl}$. As shown in Table I, the $2.1 \mathrm{mM}$ HEWL solution at $0.81 \mathrm{M} \mathrm{KCl}$ is located in the saturated region in a HEWL crystallization phase diagram, whereas the solution at $0.54 \mathrm{M} \mathrm{KCl}$ is in the soluble area. For lysozymes, the saturated solutions in which the fractal aggregates with $D$ over 1.5 are formed tend to have crystallization progress after some days [14]. In fact, some lysozyme crystals were observed in the solutions at
$0.81 \mathrm{M} \mathrm{KCl}$ after 3 days, whereas no crystals were seen in the $0.54 \mathrm{M} \mathrm{KCl}$ solutions. The F-SLS intensity increased with increasing concentrations of the added $\mathrm{KCl}$. Possible reasons for the increased F-SLS intensity include the increase in the size or the number of the lysozyme aggregates formed, and the increase in the interior density. We reasoned that the enhanced F-SLS intensity was caused by the increased density of lysozyme aggregates accompanying the size increase rather than the number of aggregates because the SLS intensity markedly increased at small angles, which exhibited the aggregate structure.

The additions of chloride salts with bivalent cations, $\mathrm{MgCl}_{2}$ and $\mathrm{CaCl}_{2}$, also enabled the formation of the fractal aggregates for pre-crystalline lysozymes. In the $2.1 \mathrm{mM}$ HEWL solution at $0.42 \mathrm{M}[4.0 \%(\mathrm{w} / \mathrm{v})] \mathrm{MgCl}_{2}$ in the saturated region, the lysozyme aggregates started to show a closely packed structure as $D$ approached the value of 1.5 in Fig. 1 (b), and likewise in the case of $\mathrm{KCl}$ addition. While the crystallization boundary concentration with $\mathrm{MgCl}_{2}$ is near $0.37 \mathrm{M}[3.5 \%(\mathrm{w} / \mathrm{v})]$ (Table I), the F-SLS pattern at the lower concentration of $0.21 \mathrm{M} \mathrm{MgCl}_{2}$ also showed a power-law type of function in Fig. 1 (b), but the estimation of $D$ gave a smaller value of 0.99 . Growth of lysozyme crystals was noticed in the solution at $0.42 \mathrm{M} \mathrm{MgCl}_{2}$ after 4 days, but no crystals were observed at $0.21 \mathrm{M} \mathrm{MgCl}_{2}$.

The F-SLS patterns for the $\mathrm{CaCl}_{2}$ additions also displayed power-law profiles showing the formation of HEWL fractal aggregates in Fig. 1 (c), but the estimated values of $D$ are lower. In particular, $D$ is as small as 1.33 even for solutions at $0.54 \mathrm{M}[6.0 \%(\mathrm{w} / \mathrm{v})] \mathrm{CaCl}_{2}$ under the saturated region. This indicates that the lysozyme aggregates at this stage had not achieved a closely packed structure of fractal aggregates that would progress to the formation of crystal nuclei. The aggregation effect of $\mathrm{Ca}^{2+}$ for lysozyme is extremely weak compared with the other cations $\mathrm{K}^{+}, \mathrm{Na}^{+}$and $\mathrm{Mg}^{2+}$, and the aggregation derived by $\mathrm{Ca}^{2+}$ would be generated at a slow speed. This explains the slowest crystal growth with $\mathrm{Ca}^{2+}$ among the other chloride salts.

The relationship between the estimated fractal dimension value and the concentration of added chloride salts is summarized in Fig. 2 for the HEWL solutions. The estimated fractal dimensions of lysozyme aggregates are

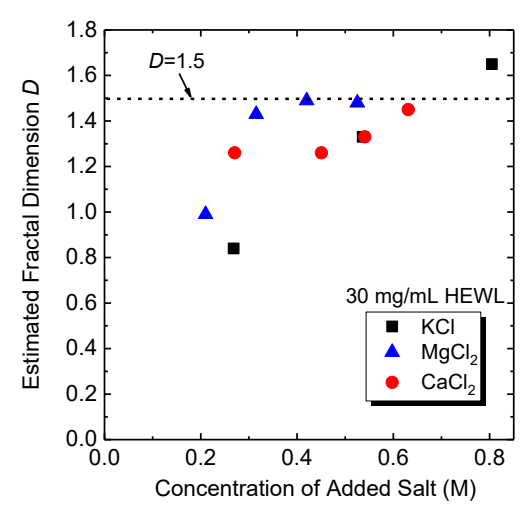

Fig.2 Dependence of fractal dimension $D$ estimated from F-SLS patterns on the concentration of salts. 
small $(<1.5)$ for solutions with any salts of $\mathrm{KCl}, \mathrm{MgCl}_{2}$ and $\mathrm{CaCl}_{2}$ in the undersaturation (soluble) regions of the crystallization phase diagram. No crystal growth of lysozymes was observed in solutions of aggregates with a loosely packed structure for $\mathrm{KCl}, \mathrm{MgCl}_{2}$ as well as that for $\mathrm{NaCl}$. In $\mathrm{KCl}$ and $\mathrm{MgCl}_{2}$ additions, the dense fractal aggregates were formed in the crystallizing solutions in the saturated regions. This relationship between the fractal dimensions of lysozyme aggregates and the crystallization conditions in the phase diagram is similar to that of $\mathrm{NaCl}$ [14].

In contrast, the estimated values of the fractal dimension are small $(<1.5)$ for $\mathrm{CaCl}_{2}$ despite the HEWL solutions being under the saturated regions. The SLS measurements were performed at about $25 \mathrm{~min}$ after mixing in the salt. This indicates that fractal aggregates with a closely packed structure had not formed in the elapsed time. Furthermore, we checked that no change in F-SLS profiles for the sample solutions with $0.54 \mathrm{M}$ $\mathrm{CaCl}_{2}$ was observed for about 3 hours after that. This indicates that the addition of $\mathrm{CaCl}_{2}$ needs a long elapsed time for forming dense fractal aggregates in precrystallization, compared to the other chloride salts. Essentially, the aggregation effect of $\mathrm{Ca}^{+}$for lysozyme is weak and the aggregation speed is slow compared with the other cations $\mathrm{K}^{+}, \mathrm{Na}^{+}$and $\mathrm{Mg}^{2+}$. This agrees well with the prediction of the aggregation effect from the Hofmeister series. Therefore, addition of $\mathrm{CaCl}_{2}$ for lysozyme crystallization is associated with the weakest aggregation effect and the latest initiation of crystal growth.

\section{CONCLUSIONS}

Aggregation effects of $\mathrm{KCl}, \mathrm{MgCl}_{2}$ and $\mathrm{CaCl}_{2}$ on the pre-crystalline lysozymes were evaluated using a smallangle FLS technique, and were associated with the crystallization conditions. The forward light scatterings revealed that like $\mathrm{Na}^{+}, \mathrm{K}^{+}$and $\mathrm{Mg}^{2+}$ induced the formation of fractal aggregates with a dense structure in precrystalline lysozyme solutions. The aggregation effect of $\mathrm{Ca}^{2+}$, however, was the slowest, giving the slowest HEWL crystal growth. The speed and structure of protein aggregation formed by adding the chloride salts are strongly related to the speed of crystal growth.

\section{Acknowledgements}

This work was supported by the Salt Science Research Foundation and partly the JSPS KAKENHI grant.

References

[1] A. McPherson, "Preparation and Analysis of Protein Crystals," John Wiley and Sons, New York (1982) pp.82-159.

[2] N. E. Chayen and E. Saridakis, Nat. Methods, 5, 147153 (2008).

[3] S. Tanaka, M. Yamamoto, K. Ito, R. Hayakawa, and M. Ataka, Phys. Rev. E, 56, R67-69 (1997).

[4] M. Muschol and F. Rosenberger, J. Chem. Phys., 103 10424-10432 (1995).

[5] S. Tanaka, K. Ito, R. Hayakawa, and M. Ataka, J. Chem. Phys., 111, 10330-10337 (1999).

[6] W. Kunz, Curr. Opin. Colloid \& Interface Sci., 15, 34-39 (2010).

[7] J. Kherb, S. C. Flores, and P. S. Cremer, J. Phys. Chem.
$B, 116,7389-7397$ (2012).

[8] T. Wakamatsu, Appl. Phys. Lett., 98 263701-(1-3), (2011).

[9] T. Wakamatsu, S. Toyoshima, and H. Shimizu, Appl. Phys. Lett., 99, 153701-(1-3) (2011).

[10] Y. Georgalis, P. Umbach, A. Zielenkiewicz, E. Utzig, W. Zielenkiewicz, P. Zielenkiewicz, and W. Saenger, J. Am. Chem. Soc., 119, 11959-11965 (1997).

[11] Y. Georgalis, A. Zouni, W. Eberstein, and W. Saenger, J. Cryst. Growth, 126, 245-260 (1993).

[12] Y. Georgalis, P. Umbach, W. Saenger, B. Ihmels, and D. M. Soumpasis, J. Am. Chem. Soc., 121, 16271635 (1999).

[13] S. Tanaka, M. Yamamoto, K. Kawashima, K. Ito, R. Hayakawa, and M. Ataka, J. Cryst. Growth, 168, 4449 (1996).

[14] T. Wakamatsu, Am. J. Anal. Chem., 5, 581-588 (2014).

[15] T. Wakamatsu, Rev. Sci. Inst., 86, 15112-(1-8) (2015).

[16] T. A. Witten and P. A. Pincus, "Structure Fluids: Polymers, colloids, surfactants," Oxford, New York, (2004) pp.54-60.

(Received January 7, 2019; Accepted March 22, 2019;

Published Online June 1, 2019) 